\title{
Perspectivas en documentación del patrimonio
}

\author{
Francisco Javier García Marco \\ Universidad de Zaragoza (España)
}

\section{Resumen}

Se analizan las aportaciones sobre documentación del patrimonio publicadas en el número 1 del volumen 11 de la revista Scire (en.-jun. 2005). Los artículos abordan el tema desde múltiples perspectivas relacionadas con la teoría y la historia de las ciencias de la documentación, los estudios bibliográficos, la aplicación de la tecnología de metadatos a la preservación de los objetos digitales, la organización del conocimiento en el campo del patrimonio y la divulgación del patrimonio bibliográfico y documental. En ellos se revindica la importancia de la documentación como actividad de transmisión del patrimonio, y su papel entre las ciencias de la memoria social. Sin desatender los retos tecnológicos de la documentación, estos estudios se concentran especialmente en sus aspectos humanísticos y sociales. La documentación aparece en ellos como un instrumento para organizar la memoria social, y, más allá, como una herramienta para rescatar del mar de la experiencia humana fragmentos de sentido capaces de iluminar el presente.

Palabras clave: Ciencias de la documentación. Patrimonio cultural. Relaciones interdisciplinares. Memoria social. Internet.

\section{Abstract}

The papers on heritage information and documentation published in Scire, volume 11, number 1 (January-June 2006), are reviewed and contextualized. The articles consider the subject from multiple perspectives related to the theory and history of Library, Archival and Information Science, bibliographic studies, metadata applied to heritage preservation, knowledge organization in heritage information, and the divulgation of heritage using the Internet. In them, the importance of information and documentation as an activity of heritage transmission is vindicated, as one of the sciences of social memory. Not withstanding the significance of the overwhelming technological challenges that Library and Information Science is facing, these studies focus in a special way on its humanistic and social aspects. Through them, Library and Information Science appears as 
an instrument to organize social memories, and, beyond that, as a tool to recover from the wide sea of human experience fragments of sense that can be relevant to enlighten the present.

Keywords: Information Science. Documentation. Cultural heritage. Social memory. Interdisciplinary relations. Internet.

\section{Introducción}

El número 1 del volumen 11 de Scire es un número monográfico dedicado a diversos aspectos relacionados con la información y documentación del patrimonio cultural desde diferentes ópticas, que reflejan la visión peculiar de archiveros, bibliotecarios y documentalistas.

Abre el número un artículo del editor (García Marco, 2005) en el que se defiende la relación estrecha de las ciencias de la documentación con el patrimonio cultural en un sentido amplio, puesto que las diferentes profesiones $-\mathrm{y}$ luego disciplinas - de la información documental han ido surgiendo precisamente para hacer posible la preservación y utilización de dicho patrimonio. En un momento en que los tecnólogos van abarcando gran parte del contenido técnico de las ciencias de la documentación y lo rebautizan con neologismos como metadatos, ontologías o repositorios que, posteriormente, son aceptados en nuestras disciplinas, los conceptos de patrimonio cultural y de memoria social pueden servir para marcar un norte que va más allá de los procedimientos y las infraestructuras, y como valores a los que anclar la supervivencia de nuestras disciplinas y profesiones.

\section{El lugar de la razón histórica en las ciencias de la documentación}

Los tres artículos que forman la primera parte del volumen tienen como denominador común su entronque metodológico, epistemológico y ontológico en las humanidades. De alguna manera, revindican el papel de las disciplinas humanísticas como fuente y destino del esfuerzo del investigador y del académico de las ciencias de la documentación, y ofrecen un interesante contrapunto a otros enfoques igualmente válidos desde la tecnología o las ciencias sociales, confirmando su carácter transversal más allá de los necesarios enmarques que exige la administración de la ciencia.

La reflexión de Manuel José Pedraza Gracia (2005) sobre el lugar de la bibliología (ciencia del libro) en las ciencias de la documentación profundiza precisamente en los avatares conceptuales de la documentación como disciplina científica a lo largo de los últimos treinta años. El artículo estudia cómo la ciencia del libro y el documento se ha ido desplazando desde el centro de la disciplina 
documental hasta un lugar cada vez más periférico en la investigación y la enseñanza dentro del área de biblioteconomía y documentación, siendo cultivado, paradójicamente, con más intensidad en otras áreas de conocimiento como la literatura o la historia. Pedraza destaca cómo al retroceso de los estudios, investigaciones y presencia académica relacionados con el libro en los estudios universitarios de biblioteconomía y documentación se contrapone el incremento de la investigación en otras áreas y la importancia que siguen teniendo las ciencias del libro en las oposiciones de acceso a bibliotecario y, por ende, en su práctica profesional. Plantea este autor cómo, en definitiva, las ciencias del libro no pueden entenderse como algo desgajado de las ciencias de la documentación, y termina enfrentándonos como colofón a la paradoja que ha ido dibujando suavemente a lo largo de todo el artículo, pues un contenido fundamental de las ciencias de la documentación está siendo desplazado del ámbito académico y científico hacia otras áreas que demuestran un interés creciente en él.

Por su parte, Agustín Vivas Moreno, Carmen Solano Macías, Aitana Martos García, Ana Isabel Aznar Castro y María Rodríguez Lairado presentan un estudio, enmarcado en la historia de la cultura, sobre "La visión de los archivos en el Antiguo Régimen hispano a través de las memorias y relatos de viajeros". A través de un trabajo pormenorizado de espigar noticias y luego de clasificarlas por temas, consiguen dibujar un panorama interesantísimo del papel de los archivos en la España moderna, a veces confirmatorio y a veces alternativo. Pero más allá del valor de estos logros, destaca la importancia del fin que se proponen: colaborar en la construcción "de una historia de la archivística de larga duración, esto es, la historia cultural de la archivística".

En el "Estado actual de los estudios bibliográficos sobre el arte en España", que cierra este bloque de tres artículos, Josefa Gallego Lorenzo analiza el panorama de los repertorios bibliográficos realizados desde el siglo XX hasta la actualidad en el campo de la historia del arte, donde han tenido tradicionalmente un gran valor instrumental. Su investigación muestra cómo esta disciplina, que un observador externo podría presumir bien dotada de instrumentos de referencia, adolece de falta de catálogos retrospectivos, solapamientos en los temas de investigación, carencia de manuales y monografías sobre materias importantes y numerosas lagunas al ignorar las publicaciones ya realizadas. Como en otras disciplinas, este estudio demuestra que el campo de la información y la documentación científica en las humanidades no es, a pesar de los esfuerzos de notables investigadores, un área en la que se produzca un esfuerzo constante y bien coordinado, lo que provoca numerosas disfunciones y una notable merma de la eficacia y la eficiencia en la investigación. He aquí, de nuevo, un gran potencial de colaboración entre las ciencias de la documentación y otras disciplinas, en este caso, las relacionadas con la historia del arte. 


\section{Los avances en normalización archivística}

La sección de estados de la cuestión de Scire presenta dos trabajos que presentan los avances que se han producido en los últimos años en normalización de la descripción archivística, uno de los aspectos fundamentales para dar acceso a la información del y sobre el pasado.

Desde su amplia experiencia, Alejandro Delgado Gómez presenta un cuidadoso análisis de los metadatos archivísticos orientados a la gestión de la conservación. Como señala el autor, a estos metadatos se les ha prestado menos atención que a los descriptivos, no obstante su carácter central en la función documental de preservar los registros, especialmente compleja en el caso de formatos mediados tecnológicamente y, concretamente, en el entorno digital. El estudio contrasta el modelo Reference Model for an Open Archival Information System (OAIS) con cinco experiencias diferentes - el proyecto Preservation Metadata for Digital Collections de la National Library of Australia (NLA), la red Networked European Deposit Library (NEDLIB), el proyecto CURL Exemplars in Digital Archives (CEDARS), el proyecto conjunto PREMIS (PREservation Metadata: Implementation Strategies) del Online Computer Library Center (OCLC) y el Research Libraries Group (RLG), y el proyecto Framework-Preservation Metadata, de la New Zealand National Library Metadata Standards - en ocho dimensiones de análisis: modularidad, extensibilidad, refinamiento, multilingüismo, interoperabilidad y reusabilidad, y existencia de una gramática formal y de una gramática formalizada. El estudio no se quedó en el mero análisis, sino que ha servido para la mejora del programa Archivo 3000 a través de tres productos: una definición de campos para un entorno de bases de datos relacionales, un formato de intercambio de registros MARC y una instancia de documento expresado en el lenguaje de marcado Extensible Markup Language (XML), mediante un XML Schema y una Document Type Definition (DTD).

En el segundo artículo, Raquel Gómez Díaz y Raquel Bringas González presentan una propuesta metodológica para el desarrollo de unos requisitos funcionales de la descripción archivística. El modelo se enmarca dentro del espíritu de la conocida iniciativa bibliográfica de sustentar la práctica descriptiva en el necesario modelo previo de las necesidades de recuperación del usuario y de sus estrategias de índole general. Con este objetivo, las autoras abordan el problema de determinar qué, para qué, por qué, cómo y para quién son las descripciones archivísticas, revisan los distintos tipos de normas que pueden servir de fuentes para resolver la cuestión - optando por las ISAD (G) como punto de referencia - y proponen chequear cada uno de sus elementos contra las funciones básicas de recuperación que pueden realizar los usuarios: identificar, gestionar, localizar y explicar. 


\section{Difusión del patrimonio e Internet}

El siguiente bloque de trabajos se centra en la utilización de las nuevas tecnologías de la información y la comunicación para la difusión del patrimonio cultural. Los dos primeros están relacionados con la difusión del patrimonio bibliográfico y documental por medio de exposiciones virtuales, y el tercero, de alcance más amplio, presenta las bases para la realización del Macrotesauro del Patrimonio Cultural-Artístico de Extremadura.

Los dos trabajos que presenta José Luis Herrera Morillas tienen como nexo el estudio de la aplicación de las tecnologías de la información y la documentación para promover el conocimiento del libro antiguo y el acceso al mismo. El primero (Herreraa, 2005a) estudia cien exposiciones virtuales de bibliotecas y propone, a partir de esa investigación, pautas y recomendaciones para su realización. Las exposiciones virtuales se han convertido en una de las actividades más importantes de las bibliotecas en la Internet, proporcionando unas enormes posibilidades de difusión a una acción cultural de gran tradición e interés. Aparte de las sugerencias técnicas, interesa ver cómo, a través del multimedia, se busca una experiencia cercana a la de la exposición presencial con el apoyo de una identidad gráfica y educativa definida. El segundo artículo (Herrera, 2005b) estudia la difusión y acceso en Internet a las encuadernaciones antiguas, tema para el que las exposiciones virtuales son, precisamente, una de las fuentes principales. Ambos estudios resaltan la importancia de libro como artefacto cultural privilegiado para la comprensión y el conocimiento del pasado, cuya materialidad resulta un elemento clave y fundamental.

En "La recuperación de la información patrimonial en el marco de la lingüística cognitiva: su aplicación a la construcción de un Macrotesauro del Patrimonio Cultural-Artístico de Extremadura”, Miguel Ángel López Alonso (2005) presenta una propuesta de diseño del Macrotesauro del Patrimonio Cultural-Artístico de Extremadura. Su propuesta se realiza desde las aportaciones de la teoría cognitiva y la lingüística del texto a la construcción de los lenguajes documentales. El trabajo continúa en la línea del pionero Tesauro de patrimonio histórico andaluz de Antonio Luis García Gutiérrez (1998a, 1998b) y del tesauro de la juventud desarrollado por el autor (López Alonso, 2007). En consonancia con esos trabajos previos y otros, el autor concluye que (2005) "un lenguaje documental, para que pueda ser capaz de relacionar sistemas de información sobre el patrimonio, debe ser integrador y servir para toda la gran variedad de aspectos del ámbito del patrimonio; no limitado a la catalogación de solo objetos de museo o solo documentos; debe poder catalogar cualquier tipo de material patrimonial material o inmaterial; ser suficientemente amplio; y derivar su utilidad como instrumento terminológico idóneo del cumplimiento de los estándares de los modernos tesauros conceptuales".

Scire. 11 : 1 (en.-jun. 2005) 11-19. ISSN 1135-3761. 


\section{Recursos: experiencias y propuestas}

El último bloque del monográfico contiene trabajos que abordan cuatro ejemplos concretos de sistemas de información y documentación de gran significación para el estudio del mundo de la cultura.

El primero de ellos es un estudio de María F. Sánchez Hernández y Yolanda Martín González (2005), en el que, en la línea de estudios anteriores, analizan ocho unidades de información europeas de referencia en el tema de la mujer y los estudios de género. Específicamente, estudian el número y el alcance de las fuentes de información que proporcionan a los usuarios, sus sistemas de clasificación, la organización y tratamiento documental de sus fondos bibliográficos, la utilización de las tecnologías de la información y su repercusión en la difusión digital de sus colecciones.

En el segundo trabajo, titulado "Necesidad del Centro de Documentación Fotográfica Nacional”, de Juan Carlos Marcos Recio, Belén Fernández Fuentes y Juan Miguel Sánchez Vigil (2005), tiene como objetivo llamar la atención sobre la necesidad de que se cree un centro de documentación fotográfica a nivel nacional en el que se incluya (para su organización y conservación) toda la documentación de este tipo existente y dispersa en diversas instituciones. Para ello, parten del estudio de los archivos fotográficos ya conocidos y organizados, de sus fondos y sus modos de trabajo, así como de las instituciones que generan o conservan documentos fotográficos. Sin duda, existe una necesidad objetiva de articular en una red el conjunto de esfuerzos que se están realizando en el campo de la preservación del patrimonio fotográfico - gran parte de ellos de alcance local - y de coronar esa red con un centro nacional de referencia que les dé soporte y visibilidad, y que pueda coordinar las políticas y los proyectos en ese campo, que, por otra parte, está recibiendo importantes apoyos de los programas de fomento de la sociedad de la información de la Unión Europea.

En el tercer trabajo, "Reflexiones, metodología, propuestas y experiencias sobre información y documentación del patrimonio documental en la universidad”, María Teresa Munera Torres (2005) reflexiona, en primer lugar, sobre el concepto de patrimonio documental, a partir de diversas definiciones, y, en especial, de la de la UNESCO. Seguidamente explora las relaciones entre los conceptos de conservación del patrimonio, universidad y educación superior. En su reflexión, la universidad aparece como generadora de "una gran cantidad de conocimiento para el beneficio y provecho de la sociedad y un abundante acopio de todo su producto intelectual, científico, cultural, administrativo y artístico desde el momento de su origen hasta la actualidad, el cual se convierte en evidencia de su función y proyección a la comunidad". Efectivamente, si la universidad es una institución responsable de transmitir, crear y difundir el conocimiento, el asegu- 
rar la conservación del patrimonio generado en el desarrollo de esa actividad aparece como una necesidad ineludible. Desgraciadamente, las universidades crean cantidades ingentes de documentación que, muchas veces, y debido a la ausencia de políticas firmes, se pierden irremisiblemente. María Teresa Munera ilustra sus ideas en la práctica a través un proyecto de investigación pionero que está llevando a cabo en su universidad, titulado "Patrimonio Documental Universidad de Antioquia, 200 años".

Por fin, en "La difusión del patrimonio histórico cultural de las casas-museo a través de los servicios ofertados por sus centros de documentación", Yolanda Martín González y Ana Belén Ríos Hilario (2005) analizan el papel que juegan las casas-museo castellano-leonesas en la difusión del patrimonio histórico cultural que albergan. Estas instituciones salvaguardan la memoria de algún personaje ilustre o vinculado de manera especial a la localidad donde se ubican, y suelen atesorar un valioso fondo documental reunido con frecuencia por el homenajeado. Partiendo de la información disponible en la red, la bibliográfica y la recogida in situ, se realiza una valoración de las distintas actividades que desarrollan y que se pueden resumir del siguiente modo: servicios y actividades típicamente museísticos - exposiciones, conferencias, etc. - ; servicios propiamente documentales - fondos constituidos, por lo general, por el legado bibliográfico de la personalidad, publicaciones propias, congresos y asambleas celebradas sobre distintos aspectos pero siempre con un eje común que es el propio homenajeado-; y servicios de apoyo a la investigación.

\section{Conclusiones}

En definitiva, esta monografía, compuesta de diversos trabajos que abordan el problema desde enfoques, metodologías e intereses distintos, revindica la importancia de la documentación como actividad de transmisión del patrimonio, y su papel fundamental entre las ciencias de la memoria social.

Esa labor debe realizarse, lógicamente, en el marco de la nueva infraestructura de transmisión del conocimiento, esto es, los ordenadores digitales y las redes de telecomunicación - fundamentalmente la Internet - , y con el usual compromiso con el desarrollo de estándares y soluciones cooperativas.

Sin embargo, contemplar la documentación desde y para el patrimonio compensa los enfoques que abordan la infraestructura tecnológica y el esfuerzo normativo como fines en sí mismos, y los reubica en su papel de medios que sirven a los objetivos y valores que hacen deseable la preservación del camino recorrido por la humanidad y sus culturas a lo largo de los siglos; un camino fascinante, lleno de recodos y bifurcaciones, y que, en cualquier caso, recorre un paisaje cargado en sí mismo de sentido. 
Desde este punto de vista, la documentación es, en lo más inmediato, un instrumento para organizar la memoria social y bucear en ella a partir de un conjunto de claves. Pero, sobre todo y más allá, constituye una herramienta para ayudar a rescatar con efectividad y eficacia fragmentos de sentido del profundo e inmenso mar de la experiencia humana, generando destellos con los que iluminar el presente, construir panorámicas de la realidad, encarar y dirigir el futuro, y recorrer - pletóricos de significados y relaciones - cada trecho del infinito camino.

\section{Referencias}

Delgado Gómez, Alejandro (2005). Archivos y metadatos de preservación: estado del arte y propuesta metodológica. // Scire: Representación y Organización del Conocimiento. 11:1 (en.-jun. 2005).

Gallego Lorenzo, Josefa (2005). El panorama actual de la producción de bibliografías de arte en España. // Scire: Representación y Organización del Conocimiento. 11:1 (en.-jun. 2005).

García Gutiérrez, A. L. (1998a). Principios de lenguaje epistemográfico: la representación del conocimiento sobre patrimonio histórico andaluz. [Sevilla], Instituto Andaluz del Patrimonio Histórico.

García Gutiérrez, A. (1998b). Tesauro de patrimonio histórico andaluz: versión 0. Sevilla, Instituto Andaluz del Patrimonio Histórico, 1998.

García Marco, Francisco Javier (2005). Ciencias de la documentación, patrimonio cultural y revolución digital: definiendo el futuro más allá de la tecnología. // Scire: Representación y Organización del Conocimiento. 11:1 (en.-jun. 2005).

Gómez Díaz, Raquel; Bringas González, Raquel (2005). El intercambio de información en el contexto de las nuevas tecnologías: normalización y requisitos funcionales aplicados a la descripción archivística. // Scire: Representación y Organización del Conocimiento. 11:1 (en.-jun. 2005).

Herrera Morillas, José Luis (2005). Encuadernaciones antiguas: normas de conservación y sistemas de información y acceso en Internet. // Scire: Representación y Organización del Conocimiento. 11:1 (en.-jun. 2005).

Herrera Morillas, José Luis (2005). Las exposiciones virtuales: un nuevo sistema de difusión cultural para las bibliotecas. // Scire: Representación y Organización del Conocimiento. 11:1 (en.-jun. 2005).

López Alonso, Miguel Ángel (2005). La recuperación de la información patrimonial en el marco de la lingüística cognitiva. // Scire: Representación y Organización del Conocimiento. 11:1 (en.-jun. 2005).

López Alonso, Miguel Ángel; Instituto de la Juventud (España) (2007). Tesauro de juventud para centros de información juvenil. Madrid, Instituto de la Juventud. Ministerio de Trabajo y Asuntos Sociales, 2007.

Marcos Recio, Juan Carlos; Fernández Fuentes, Belén; Sánchez Vigil, Juan Miguel (2005). Necesidad del Centro de Documentación Fotográfica Nacional. // Scire: Representación y Organización del Conocimiento. 11:1 (en.-jun. 2005). 
Martín González, Yolanda; Ríos Hilario, Ana Belén (2005). La difusión del patrimonio histórico cultural de las casas-museo a través de los servicios ofertados por sus centros de documentación. // Scire: Representación y Organización del Conocimiento. 11:1 (en.-jun. 2005).

Munera Torres, María Teresa (2005). Patrimonio documental en la universidad. // Scire: Representación y Organización del Conocimiento. 11:1 (en.-jun. 2005).

Pedraza Gracia, Manuel José (2005). Las ciencias del libro en el universo de la documentación. // Scire: Representación y Organización del Conocimiento. 11:1 (en.-jun. 2005).

Otlet, Paul (1934). Traité de documentation: le livre sur le livre: théorie et pratique, Bruselas: Mundaneum, 1934. Hay traducción española: Tratado de documentación: el libro sobre el libro: teoría y práctica. Murcia, [Universidad de Murcia], 1996.

Sánchez Hernández, María F.; Martín González, Yolanda (2005). La mujer en Europa: análisis de fuentes y unidades de información para los estudios de género. // Scire: Representación y Organización del Conocimiento. 11:1 (en.-jun. 2005).

Vivas Moreno, Agustín; Solano Macías, Carmen; Martos García, Aitana; Aznar Castro, Ana Isabel; Rodríguez Lairado, María (2005). La visión de los archivos en el Antiguo Régimen hispano a través de las memorias y relatos de viajeros: análisis de su representación y sistematización metodológica. // Scire: Representación y Organización del Conocimiento. 11:1 (en.-jun. 2005). 